\title{
HIV-Sero- prevalence trend among blood donors in North East Ethiopia.
}

\author{
Bekele Sharew ${ }^{1}$, Assefa Mulu², Brhanu Teka ${ }^{1}$, Tigabu Tesfaye ${ }^{3}$
}

1. Department of Medical Laboratory Sciences, Wollo University, Dessie, Ethiopia P.O. Box: 1145

2. Pharmacy department, Wollo University, Dessie, Ethiopia P. O. Box: 1145

3. Dessie Blood Bank, Dessie, Ethiopia P. O. Box: 510

\section{Emails:}

Bekele Sharew: bsharew@gmail.com(Tel: +251911165431)

Assefa Mulu: mulubaye@gmail.com(Tel: +251910980410)

Brhanu Teka: btht65@gmail.com(Tel: +251913500065)

Tigabu Tesfaye: tigabuburuk22@gmail.com(Tel: +251913877533)

\begin{abstract}
Background: Although blood transfusion is one of the known therapeutic interventions that cuts across a number of clinical disciplines. It is necessary to test all intending blood donors for HIV infection before donation. The aim of this study was to determine the prevalence of HIV among blood donors at Dessie Blood Bank, Northeast Ethiopia.

Methods: A retrospective study was conducted in Dessie Blood Bank through the year 2008- 2012. Sera from blood donors were tested for the detection of Anti HIV by using $4^{\text {th }}$ generation ELISA. Data were abstracted from records and analyzed using Microsoft Excel sheet.

Results: From the total of 9384 screened blood samples collected, the prevalence of HIV in blood donors in the blood bank was $5.1 \%$ in the five consecutive years but the trend of HIV infection has decreased from 2008(5.2\%) to $2012(2.3 \%)$. The age groups 15-24 and 35-44 were the highest prevalence and the age group 45-54 was the lowest prevalence of HIV infection. The prevalence of HIV among female (7.9\%) was higher than in male donors (4.4\%). The trend of HIV infection was decreasing for both male and female blood donors.

Conclusion: The prevalence of HIV infections among blood donors is still high in this study setting, and needs constant monitoring to evaluate prevention and control strategies to reduce the burden of transfusion-transmissible HIV infections.

Keywords: Blood donor, HIV, seroprevalence, Ethiopia.

DOI: https://dx.doi.org/10.4314/ahs.v17i3.13

Cite as: Sharew B, Mulu A, Teka B, Tesfaye T. HIV Sero- prevalence trend among blood donors in NorthEast Ethiopia. Afri Health Sci. 2017;17(3): 712-718. https://dx.doi.org/10.4314/abs.v17i3.13
\end{abstract}

\section{Background}

Although blood transfusion is one of the known therapeutic interventions that cut across a number of clinical disciplines, the practice is not without risks. HIV has continued to create a great challenge to transfusion medicine,
Corresponding author:
Bekele Sharew,
Department of Medical
Laboratory Sciences,
Wollo University, Dessie, Ethiopia
P.O. Box: 1145
Tel: +251911165431
Email: bsharew@gmail.com

especially in Africa about 10-15 \% of HIV transmission had been correlated with blood transfusions. ${ }^{1}$ Since the detection that HIV is transmitted through blood transfusion it became necessary to test all intending blood donors for HIV infection before they are transfused to ensure the safety of all blood and blood products to the recipients $^{2}$

Sub-Saharan Africa has the most serious HIV epidemic in the world. In 2012, about 25 million people were living with HIV accounting for nearly $70 \%$ of the global total. In the same year, there were an estimated 1.6 million new HIV infections and 1.2 million AIDS-related deaths. ${ }^{3}$ HIV prevalence varies significantly between regions in sub-Saharan Africa as well as individual countries. HIV prevalence in East Africa is generally moderate to high,
African

Health Sciences (a) 2017 Sharew et al; licensee African Health Sciences. This is an Open Access article distributed under the termsof the Creative commons Attribution License (https://creativecommons.org/licenses/by/4.0), which permits unrestricted use, distribution, and reproduction in any medium, provided the original work is properly cited. 
and next to Southern Africa. However, general prevalence has been in decline for the past two decades. ${ }^{3}$

In 2013 , there were an estimated 793,700 people living with HIV. HIV adult prevalence is anticipated to be $1.5 \%$ in 2011, the year in which the last Ethiopian Demographic Health Survey (DHS) was conducted. However prevalence varies according to age, sex, gender and geographical location. ${ }^{4}$

The overall seroprevalence of HIV was 3.8\% in a study conducted on blood donors at Gondar university blood bank unit ${ }^{5}$ and the peak ages for AIDS cases were 25 to 29 for both sexes. The age range at which people become infected was 15 to 24 years for females and 25 to 34 years for males. ${ }^{6}$ Among blood donors in Addis Ababa the prevalence for women was $6.9 \%$ in 1999, higher than that of men. ${ }^{7}$

On the other hand, significantly declining fashion of HIV seroprevalence were observed in the studies done in blood banks of Jimma University Specialized Hospital and Gonder University hospital ${ }^{7,8}$ and NorthWest Ethiopia. ${ }^{9}$ The Federal Ministry of Health reported the National HIV prevalence as 3.5\% and 5\% among blood donors in 2005 where the prevalence for those blood donors in the age group 15-19 years was 2.9\% but the highest prevalence occurred among donors in the age group of 30-39 years. $^{10}$

In Nigeria, the prevalence of HIV positive donors among the screened volunteers who satisfied the criteria for blood donation was $0.87 \% .{ }^{11}$ Whereas HIV incidences of $22 \%$ among blood donors in Kampala Uganda in East Africa which is significantly higher than the Nigerian studies. ${ }^{12}$

A study in India, on a rural population in 2003 reported that, the prevalence of HIV among blood donors to be $1.56 \% .{ }^{13}$ The overall seroprevalence of HIV among the total blood donors in nation wide and in Central Blood Transfusion Service (CBTS), Kathmandu, Nepal through the six years of review (from 2001-2007) was $0.33 \%$ and $0.4 \%$ respectively. ${ }^{14}$

Moreover, there is limited epidemiological data on transfusion-transmissible infections (TTI) among blood donors in Ethiopia. The magnitude of HIV among blood donors has shown variation across different settings in Ethiopia that attribute to difference in societal associated risk factors and screening methods used. ${ }^{15-19}$ The safety of blood for transfusion is a major challenge of Ethiopia, and is not well addressed. As a result, use of unsafe blood products puts the patient at risk of acquiring many T'TIs. Such patients can become potential sources of infection to the community that increase the disease burden, and can pose additional financial burden for diagnosis and treatment of these diseases. ${ }^{20,21}$ To ensure the safety and adequacy of the national blood supply in Ethiopia, mobilization and recruitment of low-risk, non-remunerated blood donors and testing of donated blood for TTIs using sensitive and specific testing methods are essential. ${ }^{22}$ Therefore, evaluating the epidemiology of TTIs can be used as indicator to assess the safety blood supply and helps to develop strategies aimed at reducing the disease burden in the communities which will ensure accessibility of safe blood supply to transfusion centers. Therefore, the aim of this study was to provide information about the trend of HIV seropositivity among the blood donors at Dessie blood bank over the study period and this would allow comparison of the seropositivity over the course of time. The finding could also be used to update intervention programs which focus on the prevention and control of HIV/AIDS.

\section{Methods}

A retrospective study was conducted at Dessie District Blood Bank during July to September 2013 by reviewing the log book's 2008-2012 data. This was a retrospective study conducted in Dessie Blood Bank Center. The study population was all blood donors who donated blood at Dessie Blood Bank from 2008-2012.The participants were those who weighed not less than $50 \mathrm{~kg}$ and were age of greater than or equal to 18 years old. A total of 9384 blood donors' records were reviewed and included in the study. Serum samples were tested for HIV using fourth generation Enzyme Linked Immunosorbent Assay (ELISA) (HIV1/2: Vironostika HIV Uni-Form II AG/ Ab, Bio-Merieux, Boxtel, Netherlands). All the tests were done following the manufacturer's instructions.

Data on socio-demographic variables, laboratory test results were collected from registration book of Dessie District Blood Bank using data extraction format. Data were cross-checked for completeness. The data was cleaned, edited and entered into computer and analyzed using Microsoft Excel sheet and the results were presented in tables and paragraph. 


\section{Ethical approval}

Ethical clearance was obtained from Wollo University Ethical Review Committee and verbal consent was obtained from Blood Bank administration before the commencement of data collection. Confidentiality of the information was ensured as codes instead of the names of the subjects were registered on the data collection format.

\section{Results}

During the 5-year period, 9,384 individuals donated blood and screened for HIV infections. From the total donors, $7514(80.1 \%)$ and $1870(19.9 \%)$ were male and female donors respectively. More men participated in the survey than women, with a male-to-female gender ratio of 4.01. The finding of this study showed that there were 476 HIV positive blood donors. Therefore, the overall prevalence of HIV infection in blood donors was found to be $5.1 \%$ in the five consecutive years (Figure 1 and Table 1).

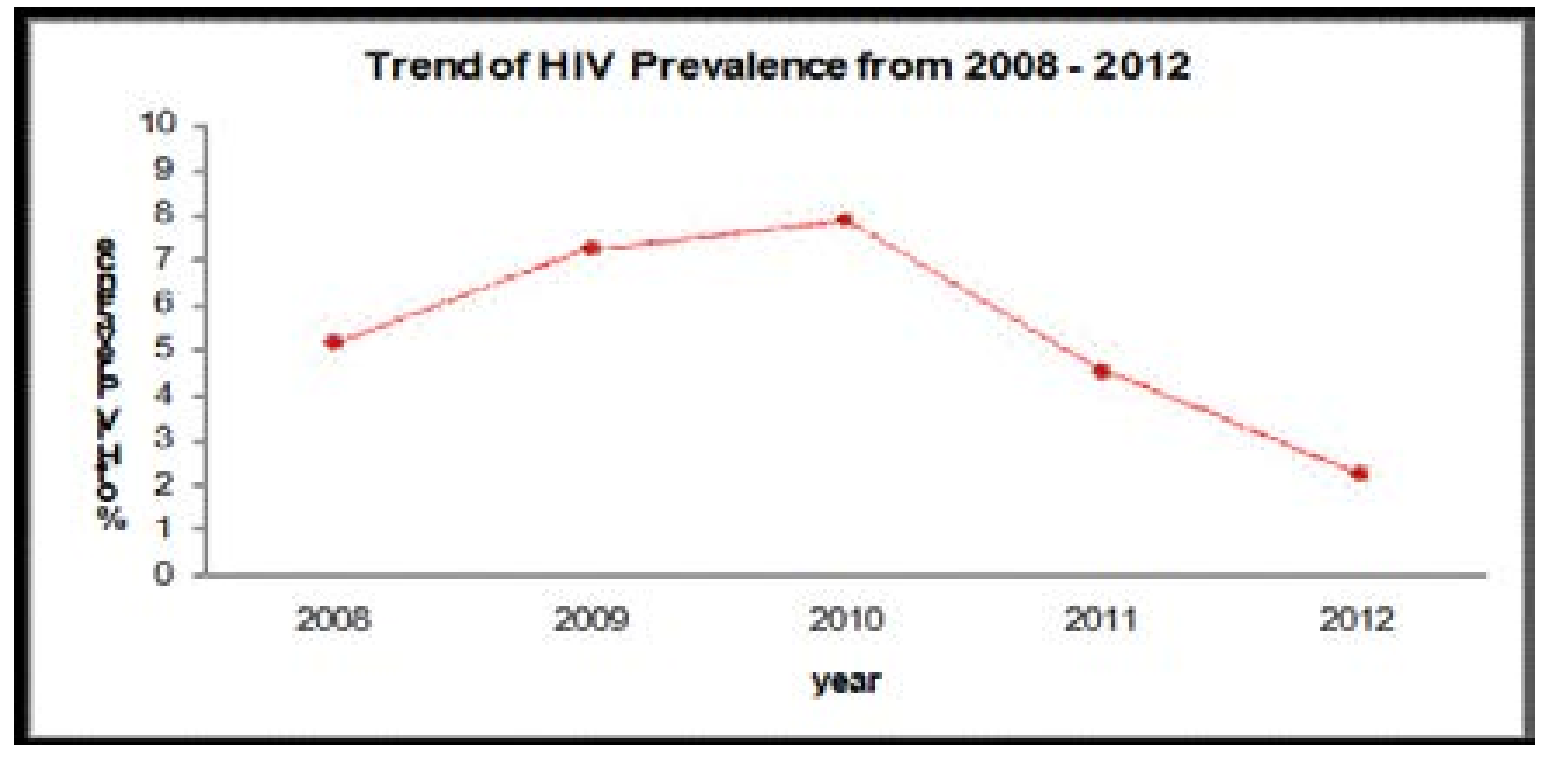

Fig.1: Trend of HIV prevalence among blood donors at Dessie Blood from 2008-2012

Table 1: Trend of HIV prevalence among blood donors at Dessie Blood Bank from 2008-2012

\begin{tabular}{|c|c|c|c|}
\hline \multirow{2}{*}{ Year } & \multirow{2}{*}{$\begin{array}{c}\text { No of Units } \\
\text { screened }\end{array}$} & \multicolumn{2}{|c|}{ HIV positive } \\
\hline & & $\mathrm{N}$ & $\%$ \\
\hline 2008 & 1432 & 75 & 5.2 \\
\hline 2009 & 1538 & 112 & 7.3 \\
\hline 2010 & 1720 & 136 & 7.9 \\
\hline 2011 & 1924 & 89 & 4.6 \\
\hline 2012 & 2770 & 64 & 2.3 \\
\hline Total & 9384 & 476 & 5.1 \\
\hline
\end{tabular}

The trend of HIV infection has decreased from 2008 to 2012. Considering age, the age groups 15-24 and 25-34 had the highest contribution and the age group 55-65 the lowest contribution of HIV infection (Table 2 and 3 ). 
Table 2: Infection with HIV among blood donors at Dessie Blood Bank by age groups from 2008 to 2012 .

\begin{tabular}{|c|c|c|c|c|c|c|c|}
\hline \multicolumn{6}{|c|}{ Year } & \multicolumn{2}{|c|}{ Total } \\
\hline group & 2008 & 2009 & 2010 & 2011 & 2012 & $\mathrm{~N}$ & $\%$ \\
\hline $15-24$ & 33 & 48 & 66 & 25 & 26 & 198 & 41.6 \\
\hline $25-34$ & 19 & 47 & 46 & 48 & 30 & 190 & 40 \\
\hline $35-44$ & 14 & 13 & 14 & 15 & 8 & 64 & 13.4 \\
\hline $45-54$ & 7 & 2 & 9 & 1 & - & 19 & 4 \\
\hline $55-65$ & 2 & 2 & 1 & - & - & 5 & 1 \\
\hline Total & 75 & 112 & 136 & 89 & 64 & 476 & 100 \\
\hline \multicolumn{8}{|c|}{ Table 3: Prevalence of HIV among age group at Dessie Blood Bank from 2008-2 } \\
\hline & Age & & Total donors & HIV & ive & Prevale & \\
\hline & $15-24$ & & 2938 & & & 6.7 & \\
\hline & $25-34$ & & 4355 & & & 4.36 & \\
\hline & $35-44$ & & 1023 & & & 6.2 & \\
\hline & $45-54$ & & 865 & & & 2.20 & \\
\hline & $55-65$ & & 206 & & & 2.43 & \\
\hline & Total & & 9384 & & & 5.1 & \\
\hline
\end{tabular}


As indicated in table 4 the prevalence of HIV among female blood donors $(7.9 \%)$ was higher than among male donors $(4.4 \%)$. The trend of HIV infection was decreasing for both male and female blood donors.

Table 4: Distribution of HIV positive blood donors by gender at dessie blood bank from 2008 -2012.

\begin{tabular}{lccccc}
\hline \multirow{2}{*}{ Year } & \multirow{2}{*}{$\begin{array}{l}\text { Total } \\
\text { donors }\end{array}$} & \multicolumn{2}{c}{ Gender } & \multicolumn{2}{c}{ HIV $(+\mathrm{ve})$} \\
\cline { 3 - 6 } & & $\begin{array}{c}\text { Male } \\
\text { Donors }\end{array}$ & $\begin{array}{c}\text { Female } \\
\text { donors }\end{array}$ & $\begin{array}{c}\text { Male } \\
\mathrm{N}(\%)\end{array}$ & $\begin{array}{c}\text { Female } \\
\mathrm{N}(\%)\end{array}$ \\
\hline 2008 & 1432 & 1194 & 238 & $54(4.5)$ & $21(8.8)$ \\
2009 & 1538 & 1201 & 337 & $76(6.3)$ & $36(10.7)$ \\
2010 & 1720 & 1373 & 347 & $98(7.1)$ & $38(11.0)$ \\
2011 & 1924 & 1502 & 422 & $61(4.1)$ & $28(6.6)$ \\
2012 & 2770 & 2244 & 526 & $40(1.8)$ & $24(4.6)$ \\
Total & 9384 & 7514 & 1870 & $329(4.4)$ & $147(7.9)$ \\
\hline
\end{tabular}

\section{Discussion}

Blood transfusion is considered as a potential risk factor for transmission of viruses which are considered to be life-threatening and have a global public health importance such as HIV. In this study, the overall prevalence of HIV infection was $476(5.1 \%)$ but showed a decreasing trend from 2008 to 2012. A decreasing trend in HIV seroprevalence among blood donors was reported from Kathmandu, Nepa ${ }^{14}$ and West African country, Mali. ${ }^{15}$

The prevalence of HIV is lower when compared with previous studies done in Ethiopia: Bahir dar, NorthWest Ethiopia $^{9}$ and Jimma, Ethiopia ${ }^{8}$. It is also lower than studies done in African countries. ${ }^{5,11,16-19,23-25}$ Our study reported higher prevalence of HIV infection compared to previous studies done in Hawassa, Ethiopia $(1.6 \%)^{15}$, Jijiga, Ethiopia $(0.1 \%)^{16}$, Iran $(0.004 \%)^{26}$, Mangalore $(0.1 \%)^{27}$, Egypt $(0 \%)^{28}$ and Jordan $(0 \%)^{29}$. Such differences in seroprevalence rate might be due to some differences in risk behaviors, geographical variation, educational programs, preventive measures, public awareness, condition of epidemic, donor selection criteria and selection procedure, sensitivity and specificity of screening technologies employed in blood transfusion centers of those countries by performance characteristics of test kits as well as diagnostic algorithms used in each study.

Sex specific prevalence of HIV infection was $4.4 \%$ for males and $7.9 \%$ for females. The difference between the two sexes was consistent with other studies that showed a higher prevalence among females. ${ }^{8,30,31}$, yet the age specific prevalence was highest among the age group 15-24 years followed by those who were $25-34$ years. The higher rate of seroprevalence in these age groups might be attributed to their being more sexually active.

\section{Conclusion}

The prevalence of HIV infections among blood donors is still high in this study setting, and needs constant monitoring to evaluate prevention and control strategies. To reduce the possible risk of infections, provision of strict criterion in recruitment of blood donors by promoting the culture of voluntary blood donations, screening of blood and blood products for these pathogens using sensitive laboratory test kits are imperious. Creating community awareness about the mode of transmission and prevention of HIV infection should be strengthened by giving health education. Moreover, conducting further community-based studies to identify societal risk factors exposing communities for blood-borne infections and developing population-specific interventions to interrupt transmission are valuable in recruiting potential volunteer non-remunerated blood donors.

\section{Authors' contributions}

TT and BS conceived and designed the study and AM and BT paid great credit to the follow up and monitoring of the data collection of the study. TT also supervised the data processing. BS and AM analyzed data. AM, BS and BT wrote the manuscript. All authors read and approved the final manuscript.

\section{Conflict of interests}

We, the authors, declare that there is no competing interest financially or non-financially. 


\section{Acknowledgement}

We are very grateful for the staff of the blood bank for their cooperation during the study. We are also thankful for Wollo University for supporting the research financially.

\section{References}

1. Fleming AF. HIV and blood transfusion in sub-Saharan Africa. Transfus sci.1997; 18(2):167-79.

2. UNAIDS. 2007 AIDS epidemic update. Available at: URL:http://www.unaids.org/en/KnowledgeCentre/HIVData/EpiUpdate/EpiUpdArchive/2007/default.asp (accessed September 2014)

3. UNAIDs, Global Report 2013 Available at: http://www.unaids.org/en/media/unaids/contentassets/documents/epidemiology/2013/gr201 UNAIDS_ Global_Report_2013_en.pdf (accessed September 2014) 4. Central Statistical Agency (Ethiopia) and ICF International. Ethiopia Demographic and Health Survey 2011. Calverton, Maryland, USA: 2012

5. Tessema B, Yismaw G, Kassu A, Amsalu A, Mulu A, Emmrich F, Sack U: Seroprevalence of HIV, HBV, HCV and syphilis infections among blood donors at Gondar University Teaching Hospital, NorthWest Ethiopia: declining trends over a period of five years. BMC infectious diseases 2010, 10(1):1.

6. Zang H, Dornadula M, Beumont $\mathrm{M}$ et al. HIV-1 in semen of men receiving slightly active ART. NEng J Med, 1998; 339: 1803-1809.

7. Kebede D, Aklilu M, Sandere E. The HIV epidemic and the state of its surveillance in Ethiopia. Ethiop Med J, 2000; 38 (4):283-302.

8. Belachew T, G/Sillasie S. Trend of HIV Sero- positivity among blood donors over 6 yrs (1995-2000) in JUSH blood bank. Ethiop J Health Sci, 2001; 11(2):97-109.

9. Kassu A, Moges F, Mekonnen F, et al. Seroprevalence of human immunodeficiency virus among blood donors in Northwest Ethiopia, 1995- 2002. Trop Doct, 2006; 36(2):106-107.

10. Federal Ministry of Health/National HIV/AIDS Prevention and Control Office. AIDS in Ethiopia. Addis Ababa: MOH sixth report. 2007.

11. Ogunkolo OF, Adenaike FA, Amballi AA, Olukoya T. Prevalence of HIV positive blood donors among screened volunteers who satisfied the criteria for blood donation in a semi-urban Nigeria population. Afr J Biotechnol. 2006. 5 (7), 553-554.
12. 12. Zachariah R, Harries AD, Nkhoma W, Arendt V, Spielmann MP, Buhendwa L, Chingi C, Mossong J. HIV prevalence and demographic risk factors in blood donors. East Afr Med J. 2002; 79: 88-91

13. Sonwane BR, Birare SD, Kulkarni PV. Prevalence of seroreactivity among blood donors in a rural population. Indian J Med.Sci. 2003; 57: 405-07

14. Tiwari BR, Karki S, Ghimire P, Yadav P, Rajkarnikar M. Prevalence of HIV in blood donors. J Nepal Health Res Counc. 2008; 6(13):93-7.

15. Birhaneselassie M: Prevalence of Transfusion-Transmissible Infections in Donors to an Ethiopian Blood Bank between 2009 and 2013 and donation factors that would improve the safety of the blood supply in underdeveloped Countries. Lab Medicine 2016, 47(2):134-139.

16. Mohammed Y, Bekele A: Seroprevalence of transfusion transmitted infection among blood donors at Jijiga blood bank, Eastern Ethiopia: retrospective 4 years study. BMC Res Notes 2016, 9:129.

17. Bisetegen FS, Bekele FB, Ageru TA, Wada FW: Transfusion-Transmissible Infections among Voluntary Blood Donors at Wolaita Sodo University Teaching Referral Hospital, South Ethiopia. Canadian Journal of Infectious Diseases and Medical Microbiology Volume 2016.

18. Gelaw B, Mengistu Y: The prevalence of HBV, HCV and malaria parasites among blood donors in Amhara and Tigray regional states. Ethiop J Health Dev 2007, 22(1):3-7. 19. Dessie A, Abera B, Wale F: Seroprevalence of major blood-borne infections among blood donors at Felege Hiwot referral hospital, Northwest Ethiopia. Ethiop J Health Dev 2007,21(1):68-69.

20. Fessehaye N, Naik D, Fessehaye T: Transfusion transmitted infections-A retrospective analysis from the $\mathrm{Na}$ tional Blood Transfusion Service in Eritrea. Pan African Medical Journal 2011, 9(1).

21. Buseri FI, Muhibi MA, Jeremiah ZA: Sero-epidemiology of transfusion-transmissible infectious diseases among blood donors in Osogbo, south-west Nigeria. Blood Transfus 2009, 7(4):293-299.

22. National Blood Transfusion Services Strategy FDRoE, ministry of Health. Addis Ababa, Ethiopia: 2005.

23. Matee M, Magesa P, Lyamuya E: Seroprevalence of human immunodeficiency virus, hepatitis B and C viruses and syphilis infections among blood donors at the Muhimbili National Hospital in Dar Es Salaam, Tanzania BMC Public Health 2006, 6(21).

24. Diro E, Alemu S: Blood safety \& prevalence of trans- 
fussion transmissible viral infections among donors at the Red Cross Blood Bank in Gondar University Hospital. Ethiopian Medical Journal 2008, 46(1):7-13.

25. Walana W, Ahiaba S, Hokey P, Vicar EK, Acquah SEK, Der EM, Ziem JB: Sero-prevalence of HIV, HBV and HCV among Blood Donors in the Kintampo Municipal Hospital, Ghana. British Microbiology Research Journal 2014, 4(12):1491-1499.

26. Farshadpour F, Taherkhani R, Tajbakhsh S, Tangestani MG, Hajiani G, Sharifi N, Taherkhani S, Nejadbolkhey A: Prevalence and Trends of transfusion Transmissible Viral Infections among Blood Donors in South of Iran: An Eleven-Year Retrospective Study. PLoS ONE 2016, 11(6):e0157615.

27. Ahmed Z, Umaru N, Shreesha K: Seroprevalence of
Transfusion Transmitted Infections among Blood Donors in Mangalore. Medica Innovatica 2012, 1(2):24-27. 28. Nada H, Atwa M: Seroprevalence of HBV, HCV, HIV and Syphilis Markers among Blood Donors at Suez Canal University Hospital Blood Bank. J Blood Disorders Transf 2013, 5:177.

29. Al-Gani FA: Prevalence of HBV, HCV and HIV-1, 2 infections among blood donors in Prince Rashed Ben Al-Hassan Hospital in North Region of Jordan. Int J Biol Med Res 2011, 2:912-916 PubMed

30. Tounkara A, Noumsi TG, Hengel RL. Increasing numbers of HIV infected blood donors reveal an expanding HIV-1 epidemic in Bamako, Mali. IntConf Aids. 2004; 15.

31. Central Statistic Authority. Ethiopian Demographic Health Survey (EDHS) 2005, September 2006:213-230. 